\title{
Nanotherapeutics: A Mini-Review
}

ISSN: 2576-9170

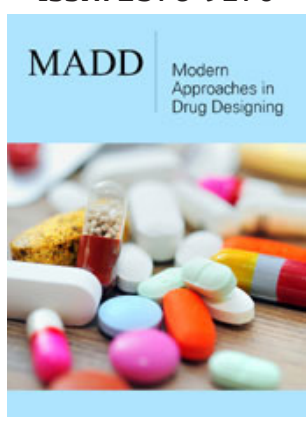

*Corresponding author: Dhimant Desai, Department of Pharmacology, Pennsylvania State University College of Medicine, Hershey-17033, PA, USA

Submission: 㭗 January 03, 2019

Published: 眥January 13, 2020

Volume 2 - Issue 5

How to cite this article: Dhanyamraju PK, Sharma A, Dovat S, Amin S, Desai D. Nanotherapeutics: A Mini-Review. Mod Appro Drug Des.2(5). MADD.000547.2020. DOI: 10.31031/MADD.2020.02.000548.

Copyright@ Dhimant Desai, This article is distributed under the terms of the Creative Commons Attribution 4.0 International License, which permits unrestricted use and redistribution provided that the original author and source are credited.

\section{Pavan Kumar Dhanyamraju ${ }^{1}$, Arati Sharma ${ }^{2}$, Sinisa Dovat ${ }^{1}$, Shantu Amin ${ }^{2}$, and Dhimant Desai ${ }^{2 *}$}

${ }^{1}$ Department of Pediatrics, Pennsylvania State University College of Medicine, Hershey, PA, USA

${ }^{2}$ Department of Pharmacology, Pennsylvania State University College of Medicine, Hershey, PA, USA

\begin{abstract}
For past few years, nanotechnology has transformed the landscape of healthcare technologies Nanotherapeutics is an application-oriented field of nanotechnology and has a wide impact in the field of medicine. The groundbreaking innovations in the field of material sciences have paved the path for nanotherapeutics, due to which nanotherapeutics have advanced remarkably and now it has become possible to deliver drugs in a target/site-specific manner. Multiple disorders like diabetes, cancer, hematological disorders, neurodegenerative and infectious diseases are the principal fields that could gain from nanotherapeutics. Also, the development of multifunctional nanotherapeutics has the potential to fill the existing gap in the current therapeutic domain. Nanotherapeutics can improve the effectiveness of the drug with minimal toxicity concerns. The development in the field of nanotherapeutics is highly impressive and has gained immense interest in pharmaceutical industries as well as in the research sector. In the present mini review, we make an attempt to highlight the recent advances in the field of nanomedicine particularly with respect to nanotherapeutics.
\end{abstract}

Keywords: Nanotechnology; Nanomedicine; Nanotherapeutics; Drug delivery; Biomedical applications

Abbreviations: Acquired Immunodeficiency Syndrome (AIDS); Acute Myeloid Leukemia (AML); Cystic Fibrosis (CF); Food and Drug Administration (FDA); Human Immunodeficiency Virus (HIV); Herpes Simplex Virus (HSV); Metastatic Breast Cancer (MBC); Magnetic Resonance Imaging (MRI); Resveratrol Loaded Lipid-Core- Nanocapsules (RSV-LNC); Severe Combined Immunodeficiency (SCID); Solid Tumor Malignancy (STM)

\section{Introduction}

Nanotechnology deals with the design, characterization, modification, investigation, production, application of devices, structures, and systems by controlling the size or shape of the objects ranging between 1-100nm in size [1]. The word "Nano" comes from the Greek word "nanos", meaning dwarf [2]. Nanomedicine is the application of nanotechnology for medical benefits. It could be defined as the utilization of nanomaterials for prevention, control, monitoring, diagnosis and treatment of diseases [3]. Nano- level quantum effects have an influence on physical, chemical, mechanical, optical and biological properties allowing scientists to take advantage of these phenomena [4]. Molecular nanotechnology is one such emerging field that could be used for the development of nanomachines also known as nano-robots. These nano-robots are fueled by xenobiotic chemical compounds and/or macromolecules of biological origin. The most interesting aspect regarding nanomaterials is that they are similar in size to many of the biological macromolecules which allow their use in in-vitro as well as in-vivo systems. A range of analytical tools, drug delivery vehicles, diagnostic kits, and physiotherapy devices have been manufactured to date through the amalgamation of nanotechnology and biological materials. Thus, the new field of nanotherapeutics has emerged showing enormous potential for research and development [1]. In comparison to traditional drug delivery approaches, in nanotherapeutics, the drug is attached to nanoparticles which helps in better delivery and thus greater efficiency with fewer side effects. With the help of nanotechnology, the efficacy and safety of conventional drugs can be improved $[5,6]$. Considering these advantages multinational pharmaceutical industries, as well as research agencies, are investing to generate gene therapies, in-vivo imaging techniques, and novel drug delivery methods. According to a new report by Grand View Research, Inc., the nanomedicine market is expected to hit $\$ 350.8$ billion by 2025 , showing a significant impact on the global 
economy $[1,7]$. Currently, the health care industry is facing multiple challenges from treating serious diseases like cancer, diabetes, cardiovascular diseases, human immunodeficiency virus (HIV)/ acquired immunodeficiency syndrome (AIDS) for providing low cost, high-quality treatment. Furthermore, treating infectious diseases with antimicrobial agents and the development of drug resistance has been one of the foremost challenges [1]. Target specificity is very crucial to achieve therapeutic efficiency and is the major hurdle with conventional drugs. Nanotherapeutics on the other hand display higher bioavailability, biocompatibility, better pharmacokinetic properties, more specific drug delivery, and targeting. The Food and drug administration (FDA) have approved many nanotherapeutics for the treatment of cancer, cardiovascular disease, autoimmune diseases, neurological diseases, Parkinson's disease, high cholesterol and diabetes in the past two decades [1,8]. Additionally, several nanocarrier based products are presently in various phases of preclinical and clinical development [9]. Diverse nanocarriers like nanogels, polymeric nanoparticles, nanoemulsions, nanocapsules, nanostructured lipid carriers, polymer micelles, dendrimers, quantum dots, nanotubes, and nanosponges are used in the development of nanotherapeutics.

\section{Applications of Nanotherapeutics}

Amikacin is a liposomal aminoglycoside antibiotic used for the treatment of multidrug-resistant Mycobacterium tuberculosis as well as gram-negative bacteria. Arikayce a modified formulation of amikacin is designed as inhalation medicine for Cystic fibrosis (CF) patients with drug-resistant pseudomonal infections $[10,11]$. Lipoquin and pulmaquin are liposomal formulations of Ciprofloxacin for the treatment of chronic pulmonary infections [12]. Doxil a liposomal formulation of doxorubicin is used in ovarian cancer, Kaposi's sarcoma, and metastatic breast cancer (MBC) [12]. Pegasys and Peglntron are pegylated interferon $\alpha-2 \mathrm{a}$ and interferon $\alpha-2 \mathrm{~b}$ used in treating hepatitis B and C [12]. Marquibo a liposomal formulation of vincristine is used for the treatment of adult acute myeloid leukemia (AML) [12]. NC-4016 is a polymeric micelle of oxaliplatin presently in phase-I clinical trials for the treatment of advanced solid tumor malignancies (STMs) [12]. NK-015 is a polymeric micelle of paclitaxel currently in phase-III clinical trials used for treating MBC [12]. NANOefavirenz (Efavirenz) and NANOlopinavir (Lopinavir) are both polymeric formulations targeting HIV and are in phase-I clinical trials [12]. Vivagel is a dendrimer-based formulation for the treatment of HIV and herpes simplex virus (HSV) [13-15]. Exparel is a pegylated adenosine deaminase that has been approved for severe combined immunodeficiency (SCID) [12]. For the treatment of advanced pancreatic, lung and breast cancers albumin-bound paclitaxel known as Abraxane is given [12]. Ferumoxsil is a siloxane coated iron oxide used as an oral contrast agent [12]. Amphotec, Abelcet and Ambisome are liposomal Amphotericin-B used in treating fungal infections [12]. Nyotran is a liposomal formulation for fighting fungal infections and currently in phase-III clinical trials [12]. Nanoplatin and NC-4016 are both polymeric micelles in phaseIII and phase-I for advanced STMs [12]. CRLX-101 is a cyclodextran conjugated formulation in phase-II clinical trials for rectal cancers
[12]. Cyclodextrin-nanosponges, as well as conjugated dendrimers, are used to enhance drug delivery in prostate cancer and pancreatic cancer respectively $[16,17]$. QA-PEI, a polymeric formulation of ammonium polyethyleneimine is in phase-II clinical trials and used for treating mixed oral flora [12]. Bismuth nanoparticles are used to concentrate radiation in radiation therapy for cancer treatment [18]. Nanoparticles containing interleukins upon reaching a tumor, release the drug thereby causing the T-cells to reproduce [18]. Preprogrammed nanorobots could be used to repair specific diseased cells [19]. Gold nanoparticles and carbon nanotubes are used in medical devices to detect oral cancers. Bacteria, viruses and other microscopic components of blood can be detected with the use of silver nanorods based devices. Quantum dots used in magnetic resonance imaging (MRI) produce high contrast exceptional quality images and very useful in imaging devices [20]. Simion et al. [21] in 2016 formulated dexamethasone loaded nanoemulsions to decrease vascular inflammation [21]. This formulation was shown to be functional in both in vitro and in vivo studies. Restasis, Diprivan, Liple, Limethason and Norvir are some examples of nanoemulsions [12]. Resveratrol loaded lipid -core- nanocapsules (RSV-LNC) has been shown to target colon cancer cells. However, further investigations are needed to validate them [22].

\section{Conclusion and Future Perspective}

The field and scope of nanotechnology have grown tremendously in the last two decades. In particular, nanomedicine has achieved many breakthroughs and has become an indispensable part of modern medicine. Also, the availability of multiple nanocarriers has boosted the development of various nanotherapeutics. In comparison to conventional chemotherapeutics, nanotherapeutics hold better pharmacokinetic and bioavailability profiles. In the near future, nanomedicine/nanotherapeutics will take us a step closer to personalized medicine. Furthermore, with advances in nano-diagnostic tools, the specificity and sensitivity of diagnostic tests have greatly improved. While the FDA has approved several nanotherapeutics for treating myriad diseases, however there are still problems to be addressed, for example, nanotherapeutics suffer from issues like inadequate tissue distribution, instability in circulation, and safety. Still much more remains to be done to achieve the ultimate goal of efficient, effective, precise, safe and specific treatment through nanotherapeutics.

\section{Acknowledgment}

Departments of Pharmacology and Pediatric, Penn State Cancer Institute, and Four Diamonds Fund of Pennsylvania State University College of Medicine, Hershey, PA -17033, USA

\section{Conflict of Interest}

None.

\section{References}

1. Prasad M, Lambe UP, Brar B, Shah I, Ranjan K, et al. (2018) Nanotherapeutics: An insight into healthcare and multi-dimensional applications in medical sector of the modern world. Biomed Pharmacother 97: 1521-1537. 
2. Bharali DJ, Mousa SA (2010) Emerging nanomedicines for early cancer detection and improved treatment: Current perspective and future promise. Pharmacol Ther 128(2): 324-335.

3. Tinkle S, McNeil SE, Muhlebach S, Bawa R, Borchard G, et al. (2014) Nanomedicines: Addressing the scientific and regulatory gap. Ann N Y Acad Sci 1313: 35-56.

4. Seigneuric R, Markey L, Nuyten DS, Dubernet C, Evelo CT, et al. (2010) From nanotechnology to nanomedicine: Applications to cancer research. Curr Mol Med10(7): 640-652.

5. Wagner V, Dullaart A, Bock AK, Zweck A (2006) The emerging nanomedicine landscape. Nat Biotechnol 24(10): 1211-1217.

6. Freitas RA (2005) What is nanomedicine? Nanomedicine 1(1): 2-9.

7. (2017) Nanomedicine Market Analysis by Products T, Regenerative Medicine, Diagnostics), By Application, (Clinical Oncology, Infectious diseases), By Nanomolecule (Gold, Silver, Iron Oxide, Alumina), \& Segment Forecasts, 2018 - 2025. Nanomedicine Market Analysis by Products, (Therapeutics, Regenerative Medicine, Diagnostics), By Application, (Clinical Oncology, Infectious diseases), By Nanomolecule (Gold, Silver, Iron Oxide, Alumina), \& Segment Forecasts, 2018 - 2025.

8. Bhaskar S, Tian F, Stoeger T, Kreyling W, de la Fuente JM, et al. (2010) Multifunctional Nanocarriers for diagnostics, drug delivery and targeted treatment across blood-brain barrier: perspectives on tracking and neuroimaging. Part Fibre Toxicol 7: 3.

9. Webster TJ (2008) Projections for nanomedicine into the next decade: but is it all about pharmaceuticals? Int J Nanomedicine 3(1): i.

10. Caminero JA, Sotgiu G, Zumla A, Migliori GB (2010) Best drug treatmen for multidrug-resistant and extensively drug-resistant tuberculosis. Lancet Infect Dis 10(9): 621-629.

11. Canton R, Cobos N, de Gracia J, Baquero F, Honorato J, et al. (2005) Antimicrobial therapy for pulmonary pathogenic colonisation and infection by Pseudomonas aeruginosa in cystic fibrosis patients. Clin Microbiol Infect 11(9): 690-703.

12. CasterJM, PatelAN,ZhangT,Wang A(2016) Investigationalnanomedicines in 2016: A review of nanotherapeutics currently undergoing clinical trials. Wiley Interdiscip Rev Nanomed Nanobiotechnol 9(1).
13. O'Loughlin J, Millwood IY, McDonald HM, Price CF, Kaldor JM, et al. (2009) Safety, tolerability, and pharmacokinetics of SPL7013 gel (VivaGel): A dose ranging, phase I study. Sex Transm Dis 37(2): 100-104.

14. Price CF, Tyssen D, Sonza S, Davie A, Evans S, et al. (2011) SPL7013 Gel (VivaGel(R)) retains potent HIV-1 and HSV-2 inhibitory activity following vaginal administration in humans. PLoS One 6(9): e24095.

15. Cohen CR, Brown J, Moscicki AB, Bukusi EA, Paull JR, et al. (2011) A phase I randomized placebo-controlled trial of the safety of 3\% SPL7013 Gel (VivaGel(R)) in healthy young women administered twice daily for 14 days. PLoS One 6(1): e16258.

16. Gigliotti CL, Minelli R, Cavalli R, Occhipinti S, Barrera G, et al. (2016) In vitro and in vivo therapeutic evaluation of camptothecin-encapsulated beta-cyclodextrin nanosponges in prostate cancer. J Biomed Nanotechnol 12(1): 114-27.

17. Ozturk K, Esendagli G, Gurbuz MU, Tulu M, Calis S (2016) Effective targeting of gemcitabine to pancreatic cancer through PEG-cored Flt-1 antibody-conjugated dendrimers. Int J Pharm 517(1-2): 157-167.

18. Sebastian R (2017) Nanomedicine-the future of cancer treatment: A review. J Cancer Prev Curr Res 8(1): 00265.

19. Loo C, Lin A, Hirsch L, Lee MH, Barton J, et al. (2004) Nanoshell-enabled photonics-based imaging and therapy of cancer. Technol Cancer Res Treat 3(1): 33-40.

20. Kirti Vishwakarma (2012) A brief review on role of nanotechnology in medical sciences. Proceedings of All India Seminar on Biomedical Engineering 2012 (AISOBE 2012), Springer, India, pp.53-63.

21. Simion V, Constantinescu CA, Stan D, Deleanu M, Tucureanu MM, et al. (2016) Selectin targeted dexamethasone-loaded lipid nanoemulsions: A novel therapy to reduce vascular inflammation. Mediators Inflamm, 1625149.

22. Feng M, Zhong LX, Zhan ZY, Huang ZH, Xiong JP (2017) Enhanced antitumor efficacy of resveratrol-loaded nanocapsules in colon cancer cells: Physicochemical and biological characterization. Eur Rev Med Pharmacol Sci 21(2): 375-382. 